\title{
Managing Patients with Insulin Pumps and Continuous Glucose Monitors in the Hospital: to Wear or Not to Wear
}

\author{
Tiffany Yeh ${ }^{1} \cdot$ Michele Yeung ${ }^{1} \cdot$ Felicia A. Mendelsohn Curanaj ${ }^{1}$ \\ Published online: 15 January 2021 \\ (C) The Author(s), under exclusive licence to Springer Science+Business Media, LLC part of Springer Nature 2021
}

\begin{abstract}
Purpose of Review As the prevalence of diabetes mellitus in the USA continues to rise, so does the popularity of diabetes management devices such as continuous glucose monitors (CGMs) and insulin pumps. The use of this technology has been shown to improve outpatient glycemic outcomes and quality of life and oftentimes may be continued in the hospital setting. Our aim is to review the current guidelines and available evidence on the continuation of insulin pumps and CGMs in the inpatient setting.

Recent Findings Patients with diabetes are at higher risk for hospitalizations and complications due to hyper- or hypoglycemia, metabolic co-morbidities, or as seen recently, more severe illness from infections such as SARS-CoV-2. The maintenance of euglycemia is important to decrease both morbidity and mortality in the hospital setting. There is consensus among experts and medical societies that inpatient use of diabetes technology in carefully selected patients with proper institutional protocols is safe and can improve inpatient glycemic outcomes and reduce hypoglycemia. During the COVID-19 pandemic, CGMs played a vital role in managing hyperglycemia in some hospitalized patients.

Summary Insulin pumps and CGMs have the potential to transform glycemic management in hospitalized patients. In order for institutions to safely and effectively incorporate these technologies on their inpatient units, hospital-based providers will need to be able to understand how to manage and utilize these devices in their practice in conjunction with diabetes experts.
\end{abstract}

Keywords Insulin pump · Continuous glucose monitor · Hybrid closed-loop · Covid-19 Inpatient diabetes management . Diabetes technology

\section{Introduction}

An estimated 1 in every 10 adults in the USA has diabetes mellitus, and the prevalence continues to rise alongside the rates of obesity. Patients with diabetes are at higher risk for hospitalizations and complications due to hyper- or hypoglycemia, metabolic co-morbidities, or as seen recently, more

Tiffany Yeh and Michele Yeung Both authors contributed equally in the writing of this paper, and are listed alphabetically

This article is part of the Topical Collection on Hospital Management of Diabetes

Tiffany Yeh

tiy9005@med.cornell.edu

1 Department of Medicine, Division of Endocrinology, Diabetes, and Metabolism, Weill Cornell Medicine / New York Presbyterian Hospital, 1305 York Avenue, 4th Floor, New York, NY 10021, USA severe illness from infections such as SARS-CoV-2. Once hospitalized, glycemic goals outlined by the American Diabetes Association [1] help to improve clinical outcomes and reduce complications during the admission. Thus, the importance of maintaining euglycemia in both the outpatient as well as the inpatient setting is paramount to the health of adults with diabetes.

Fortunately, in the past decade, technology has grown exponentially to aid in the management of patients with diabetes. The use of continuous subcutaneous insulin infusion (CSII or insulin pumps) and continuous glucose monitoring systems (CGMs) has greatly improved the care and quality of life for patients and is widely used in the ambulatory setting. In 2018, an estimated $30-40 \%$ of patients with type 1 diabetes utilized some type of insulin pump or CGM [2]. Increasingly, this technology follows the patient into the hospital setting, and hospital-based healthcare providers will need to understand how to manage and utilize these devices in conjunction with diabetes experts. In April of 2020, The Continuous Glucose 
Monitors and Automated Insulin Dosing Systems in the Hospital Consensus Guideline Panel convened and developed recommendations for clinicians, as well as guidelines that should be followed to build an environment for facilitating safe use of these devices [3]. This review will discuss current guidelines and studies on the use of insulin pumps and CGMs in the inpatient setting.

\section{Background}

Insulin pumps are continuous subcutaneous insulin infusion (CSII) devices that deliver a continuous infusion of rapid acting insulin subcutaneously. They provide a preset variable rate over $24 \mathrm{~h}$ per day to mimic "basal" insulin delivery, which is the amount of insulin the patient requires to maintain euglycemia when not eating. There is also a "bolus" feature which is programmed to calculate an additional dose of insulin based on preset insulin-to-carbohydrate ratios and insulin sensitivity factors. This provides insulin to cover food intake and correct hyperglycemia. Typically, the device consists of a control unit (the pump itself), a reservoir of insulin, and an insertion site that delivers insulin via a subcutaneous cannula. The control unit may or may not be connected to the reservoir/ insertion site with tubing. A patch insulin pump is a type of CSII device that does not have tubing, and consists of a pod of insulin attached to the body, with a remote control unit. The primary benefit of an insulin pump is the avoidance of multiple subcutaneous insulin injections per day and has been shown to decrease hemoglobin $\mathrm{A} 1 \mathrm{c}$ and increase time in range (BG 70-180 mg/dL) [2].

Continuous glucose monitors are devices that measure subcutaneous interstitial glucose levels throughout the day. Most communicate wirelessly to a receiver or phone that displays the current interstitial glucose and recent trend of sensor glucose values. Some have predictive alarms for hypo- or hyperglycemia, which allow patients the opportunity to take action before an event occurs. CGMs have also been shown to reduce hemoglobin A1c and increase time in range [2].

Of note, it is important to be aware that both insulin pumps and CGMs are not completely automated systems, and patients and providers need to be educated and comfortable with basal/bolus insulin therapy, carbohydrate counting, prandial insulin dosing, and correcting hyper- and hypoglycemia.

\section{Inpatient Use of Insulin Pumps}

The Endocrine Society, the American Diabetes Association, the American Association of Clinical Endocrinologists, and the Association of Diabetes Care \& Education Specialists all encourage supporting patients who are physically and mentally able to continue to use their insulin pumps when hospitalized, with appropriate hospital personnel and policy guidance $[4,5]$. The goal of continued insulin pump usage during the hospital stay is to foster continued patient independence with diabetes self-care within parameters that optimize safety and efficacy [6].

Previous studies showed that maintaining glucose targets with an insulin pump is not inferior to subcutaneous insulin injections. In the largest study thus far of 253 hospitalizations, there was a lower frequency of both hyper- and hypoglycemic (defined as a BG $<40 \mathrm{mg} / \mathrm{dL}$ ) events [7, 8]. Patients reported high patient satisfaction when allowed to continue diabetes self-care in collaboration with the hospital staff, and often this can be an opportunity to adjust and optimize pump settings [7].

In 2017, the Diabetes Technology Society issued an expert panel consensus statement reviewing the safety of insulin pump use during hospitalization, stating 3 essential building blocks for safe use: (1) proper patient selection on admission, (2) establishing hospital-wide policies on pump use, and (3) effective patient-staff communication [7]. The 2017 consensus statement was updated and expanded to include automated insulin dosing (AID) systems in 2020 [3]. Errors in management of an insulin pump can result in severe hypo- or hyperglycemia that may not be caught by typical hospital safeguards, such as pharmacy review or scheduled point-of-care testing. Patient safety is paramount, so continued use of an insulin pump or CGM in the inpatient setting needs to be carefully considered.

\section{On Admission}

When a patient on an insulin pump is admitted, the decision should be made on an individual basis as to whether or not the patient can continue to safely use their pump. With appropriate patient selection on admission, established hospital policies, and staff familiar with diabetes technology, most patients can safely transition to continued use while in the hospital.

On admission, there should be documentation of the manufacturer and model of pump, type of insulin formulation, and settings such as basal rate(s), insulin-to-carbohydrate ratio(s), insulin sensitivity (correction) factor(s), dietary intake, and frequency of point-of-care glucose testing with a hospital glucose meter. Any necessary adjustments to the pump settings due to the current medical condition of the patient should be made and documented [2]. Patients should not make changes to their pump settings without first discussing with hospital staff [6]. If possible, the target glucose set within the pump should be adjusted to $140-180 \mathrm{mg} / \mathrm{dL}$, as per ADA guidelines for critically ill and non-critically ill hospitalized patients with diabetes [1]. Patients should be made aware of these targets, as it likely differs from outpatient goals [6]. If hospital policy dictates a signed patient agreement, the agreement should delineate the responsibilities of the patient to manage their 
device and include consent to share pump settings and information with hospital staff and to report any issues $[2,3,9]$.

Different insulin pump models will have different screens, buttons, and modes of delivering insulin [6]. Patients are often more familiar than most hospital staff on the operations of their specific pump. Therefore, it is important that both the patient and a member of the inpatient healthcare team are familiar with how to maneuver the pump settings and screens. Oftentimes, hospital policies dictate that an endocrinologist, diabetes care and education specialist, or inpatient diabetes team are consulted on admission of a patient wearing a CSII or CGM device.

\section{Reasons to Stop the Pump on Admission}

Inpatient diabetes self-management requires a complex skill set of pump manipulation. Patients should be able to demonstrate knowledge of the pump and possess the manual dexterity to navigate, change, and refill the pump. They should, at a minimum, be able to deliver a bolus and suspend the basal insulin delivery if needed. Patients should change their insertion site at least every $72 \mathrm{~h}$, or sooner if needed. Despite patient preference to remain on their device during hospitalization, not all patients on an insulin pump can demonstrate safe use and should strongly consider switching to subcutaneous insulin injections and re-training in the outpatient setting $[6,7]$.

Below is a sample list of contraindications to inpatient CSII [adapted from Umpierrez, et al. [2], Thompson, et al. [6], and Davis, et al. [10]]:

- Impaired level of consciousness or confusion

- Critical illness requiring intensive care

- Diabetic ketoacidosis or hyperosmolar hyperglycemic state

- Psychiatric illness or suicidal ideation

- Patient unable to use hands and/or physically manipulate pump due to medical condition

- Patient unwilling to participate in diabetes self-management, or share pump management decisions with trained hospital providers

- Lack of pump supplies or mechanical pump malfunction

- Lack of trained healthcare providers or available diabetes specialists to supervise pump therapy

- Medical team decision for health and safety of the patient

If the patient is admitted with an altered level of consciousness or confusion, they should not be continued on CSII. Suicidal ideation or attempt is a contraindication to pump use as well, as patients can intentionally harm themselves by giving too much or too little insulin [2].

In the case of an admission for diabetic ketoacidosis or hyperosmolar hyperglycemic state, the pump must be stopped, as pump malfunction can actually be a cause. A blockage or leakage in the infusion catheter, infusion set, or insulin reservoir can lead to inadequate administration of insulin. The patient should be treated as per hospital protocol. Once metabolic derangements have resolved and the patient is clinically stable, the pump may be resumed if the patient is willing, assuming any mechanical pump issues are resolved as well $[2,9]$. The patient or family need to be able to provide necessary pump supplies, such as infusion sets and insulin reservoirs, since each device model will have different components that a hospital would not typically have on formulary $[2,7]$. If supplies are not available, then pump therapy should be discontinued.

Finally, if the inpatient healthcare team is not familiar with CSII, and there is not an endocrinology or inpatient diabetes consultation service available, then continued pump use is not recommended. Lack of available expert support could lead to confusion among hospital staff, medication errors, inappropriate continuation of the pump without necessary adjustments, and potential harm to the patient $[2,6,7]$. Virtual consults for patients on pumps and CGM may be a viable solution.

\section{During Hospitalization}

With the support of the endocrinology or inpatient diabetes service, patients on CSII should be allowed to continue using their device if appropriate throughout the hospitalization. The patient's physical, cognitive, and emotional ability to selfmanage should be assessed daily throughout the hospitalization. Acute infections, certain medications, changes in dietary intake, and changes in renal function can result in changes in insulin requirements and insulin sensitivity, so regular evaluations and adjustments of pump settings are crucial [7].

A hospital blood glucose meter (BGM) should be used for calibration and dosing of insulin, as accuracy is important when using CSII. Calibration with a continuous glucose monitor will be discussed separately. Scheduled point-of-care testing throughout the day should be ordered and documented. A hospital glucose meter is calibrated daily as per regulations and ensures accurate and consistent checking of blood glucose levels by hospital staff [7].

The infusion set of a tethered CSII device includes a subcutaneous catheter made of either plastic or metal connected to a superficial insertion set with adhesive. Failure to change infusion sets at the device manufacturer's recommended intervals (commonly $48 \mathrm{~h}$ for metal, $72 \mathrm{~h}$ for plastic) can result in site infection or problems with insulin delivery. In hospitals and communities where methicillin-resistant Staphylococcus aureus (MRSA) or other multi-drug-resistant organisms are present, a site infection can result in cellulitis, abscess, or even bacteremia. Therefore, daily inspection and documentation of the site are recommended, similar to any other subcutaneous catheters. If an infection occurs, the infusion set and connected 
insulin reservoir must be discarded, and a new set in a new location must be inserted [6,9]. For patients wearing a patch insulin pump, in the setting of an infection, the patch pump unit with the insulin reservoir should be replaced completely.

Patient hand-off throughout the hospitalization is an area of much attention recently. CSII devices should be included as part of the hand-off, even for brief imaging or procedures. Procedural staff, as well as those in the peri-operative area, should be made aware of the pump. If it becomes disconnected or dislodged for any reason, there should be instructions on whether to allow reconnection or to transition to subcutaneous insulin therapy [6]. Patients with type 1 diabetes or insulindependent type 2 diabetes must receive basal insulin immediately (or in advance) if the insulin pump is suspended or removed.

\section{Reasons to Adjust or Stop the Pump During Hospitalization}

During the course of a hospitalization, a patient's medical condition may change. Similar to upon admission, should a contraindication arise, CSII should not be continued. Other situations may not necessitate stopping the insulin pump; rather, the pump settings should be adjusted (Table 1). In all situations, consider expert consultation with the endocrinology or inpatient diabetes consult team, to discuss how to safely proceed. If there is concern for safety with continued pump use, it should be discontinued, and the patient transitioned to subcutaneous insulin injections. The device can usually be resumed at discharge, given appropriate patient conditions and provider evaluation prior to resumption.

Administered medications or anesthesia may impair consciousness or cause confusion. Changes in skin perfusion (i.e., due to hypotension), blood pressure, or body temperature may impact the accuracy of CSII devices that rely on communication with a continuous glucose monitor [6]. Limited mobility or depending on hospital staff for transfer may result in pulling on the tubing and accidental dislocation of the insertion set. Renal injury or hepatic failure resulting in uremia or encephalopathy could impair the ability of the patient to operate the pump. Decreased renal function can result in decreased clearance of insulin potentially leading to hypoglycemia. Steroid use, depending on type, dose, and duration, will greatly impact glycemic outcomes and insulin sensitivity. Procedures and imaging will be discussed in a later section.

Similar to any device, insulin pumps can experience mechanical or technical failure from use or mishandling. The plastic casing of the pump or reservoir can crack, or the tubing can become kinked. The electrical components of the pump may be damaged by water. Examples of technical failure include an error message displayed in the absence of an identifiable problem, or the reverse when a critical problem occurs without an appropriate alarm (kinked tubing or infusion set, presence of air in the tubing, or low battery). Hospital staff can try to troubleshoot by calling the device support line for assistance. If the problem is unable to be resolved promptly, the pump should be discontinued [6]. Of note, in the largest published study of 253 hospitalizations, pump malfunctions are uncommon. Cook et al. noted only 1 adverse event of a kinked infusion catheter, and no site infections, mechanical failures, or episodes of diabetic ketoacidosis (DKA) [7, 8]. In a prospective study initiating insulin pumps during hospitalization, 11 pump alarms occurred and involved catheter occlusion, empty insulin reservoir, and connection errors [11].

\section{Special Considerations}

\section{Critically III Patients}

If patients are admitted to the ICU and/or are critically ill, it is not recommended to continue on CSII. Instead, they should be transitioned to intravenous (IV) insulin infusion for tight glycemic management. $[2,4,10]$. Once stabilized, insulin infusions offer an opportunity to modify basal insulin rates set in the patient's own pump.

\section{Imaging}

Precautions need to be taken with certain types of imaging or inpatient procedures (see Table 2 for details). Ionizing radiation or electromagnetic field exposure can damage the

Table 1 Common medical conditions during hospitalization that affect insulin pump management

\begin{tabular}{ll}
\hline Medical condition & Recommendation for insulin pump management \\
\hline Altered mental status, confusion or unresponsive & Stop insulin pump use and switch to MDI \\
Acute kidney injury & Decrease basal and bolus settings \\
NPO, nothing by mouth & Decrease basal rate by at least $20 \%$ \\
Decreased appetite or change in dietary habits & Decrease basal and bolus settings \\
Steroid use & Decrease insulin sensitivity factor and consider expert consultation or stopping pump \\
Weakness or impaired vision or hand strength & Evaluate pump self-management skills \\
Narcotic or anesthetic use & Evaluate pump self-management skills \\
\hline
\end{tabular}

MDI, multiple daily injections. Adapted from Thompson, et al. [6] 


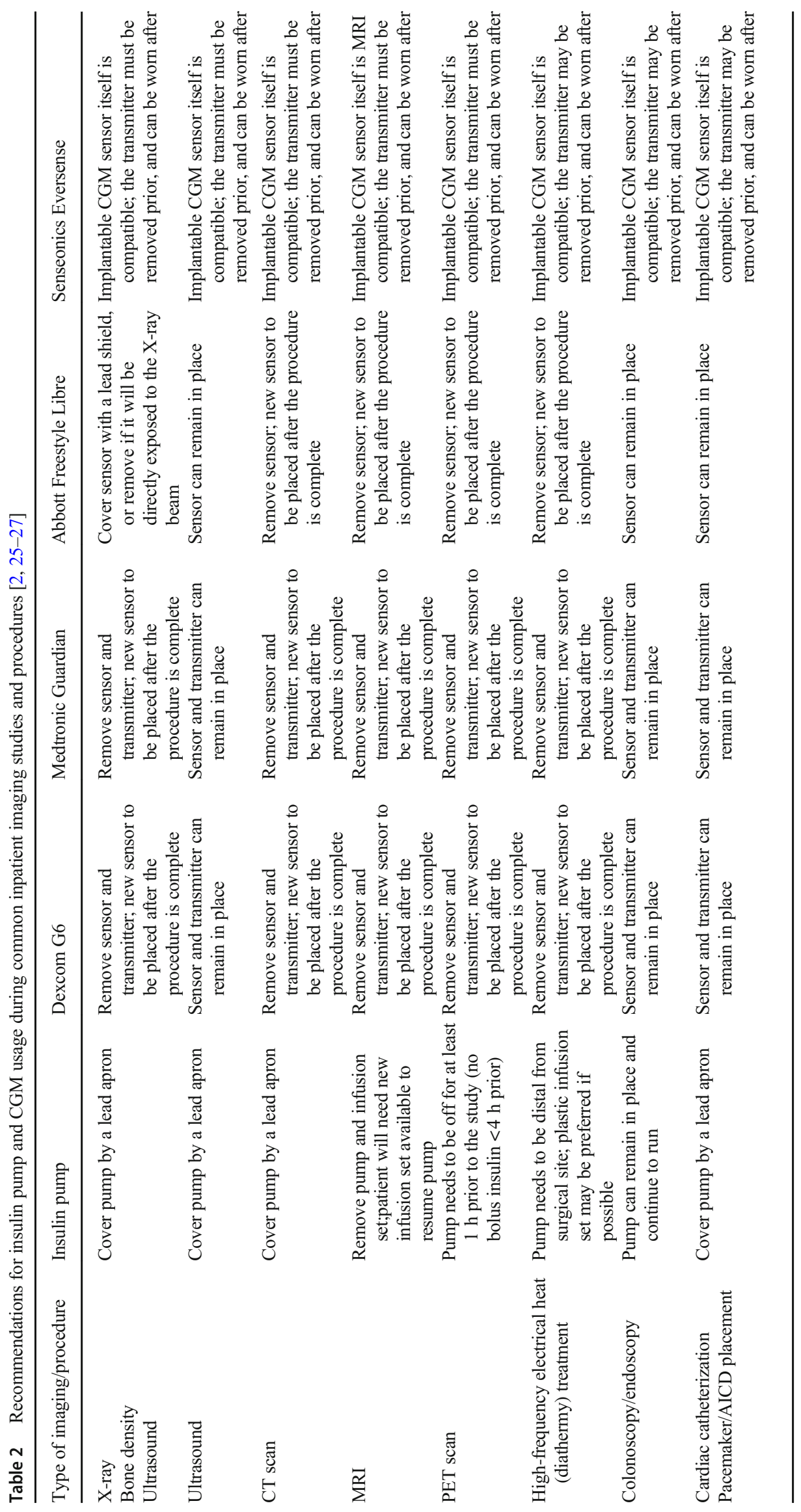


electrical components of a pump. If an imaging study or procedure is anticipated to be longer than $2 \mathrm{~h}$, and the pump is disconnected, patients should be transitioned temporarily to subcutaneous insulin therapy [6].

\section{Procedures}

If the patient needs to undergo a procedure while admitted, the pre-op decision of whether or not to continue an insulin pump will depend on the type of procedure, duration, post-operative recovery time, and whether there will be exposure to an electromagnetic field (including electric shock for defibrillation). For short procedures under $2 \mathrm{~h}$, patients can typically continue to wear their pumps throughout the procedure. For procedures or surgeries longer than $2 \mathrm{~h}$, switching to continuous IV insulin infusion should be considered. The infusion set and insertion site should be examined and noted, and far from the area of the procedure (see Table 2 for details). If anesthesia is involved, the anesthesiologist should be aware and have access to the insulin pump, in case it needs to be suspended or disconnected $[2,9,12]$. As mentioned above, during patient hand-off, all members of the transfer team should be aware of the insulin pump, including staff in the peri-operative or holding area [6].

\section{Hypoglycemia}

Hypoglycemia is a serious risk for patients receiving insulin therapy - either via subcutaneous insulin injection or insulin pump - and unfortunately occurs frequently in hospitalized patients [11]. It can be from multiple etiologies, but patients on CSII are especially prone to this. Due to changes in their medical conditions during admission, most commonly a decrease in food intake or impaired renal function, insulin requirements often decrease. Therefore, outpatient basal and bolus settings tend to be too high and should be adjusted during the hospitalization. If hypoglycemia is severe or recurrent, stopping the insulin pump and transitioning to subcutaneous insulin injections should be considered.

If the patient is admitted with severe hypoglycemia (confusion, loss of consciousness, seizure), the insulin pump should be stopped, and the hypoglycemia treated with dextrose. The pump could be restarted once the hypoglycemia is resolved, the cause of hypoglycemia is identified, and diabetes self-management skills are reviewed. The inpatient diabetes or endocrinology consult team should evaluate the pump settings prior to reinitiation, to ensure appropriate insulin delivery settings [9].

\section{Hyperglycemia}

Certain inpatient situations can result in inadvertent hyperglycemia, such as high-dose glucocorticoids or enteral feedings.
Infections, changes in dietary intake, and the stress of hospitalization itself can also result in hyperglycemia. In these situations, close collaboration with the inpatient diabetes team may be necessary for ongoing insulin pump setting adjustments.

\section{When Off the Pump}

As mentioned above, there are certain situations during the hospitalization in which it may no longer be safe for the patient to continue using their own insulin pump. If the decision is made to discontinue the pump, patients should be transitioned to a subcutaneous insulin injection regimen consisting of basal and bolus insulin, or continuous IV insulin infusion. The pump should be discontinued $2 \mathrm{~h}$ after the first dose of long-acting (basal) insulin is given. Administration of basal insulin is especially important in patients with type 1 diabetes who need insulin on board at all times to prevent DKA. Mealtime coverage should be given as rapid-acting insulin boluses [2]. Further discussion on inpatient management of diabetes with subcutaneous insulin is beyond the scope of this paper.

\section{Discharge Planning}

In the outpatient setting, the use of insulin pumps and CGMs has continued to show improvements in glycemic outcomes and quality of life in patients with diabetes. Therefore, if patients were on CSII prior to admission, and are medically stable for discharge home, it would be ideal for them to either continue or resume their pump at discharge.

Prior to discharge, the endocrinology or inpatient diabetes consult service should check the basal and bolus insulin settings to confirm that they are appropriate, taking into consideration pre-admission insulin requirements and changes in medical conditions during hospitalization [2]. Appetite or dietary recommendations may be different at discharge, as well as a patient's activity level and potentially weight. This is a good opportunity to reassess and review diabetes selfmanagement skills and CSII knowledge.

\section{Continuous Glucose Monitors}

The use of point-of-care (POC) blood glucose (BG) testing has been the mainstay for monitoring glycemia and informing treatment of hospitalized patients with diabetes. In hospitalized patients with diabetes, POC blood glucose (BG) testing is commonly performed 3 to 4 times daily to monitor glycemic outcomes and to adjust insulin doses. However, the intermittent nature of testing and the associated time burden for nursing and ancillary hospital staff are significant limitations of POC BG testing. 
The ability of CGM to provide glucose values every 15 min, glucose trends, and alerts and alarms predicting dysglycemia allows for a more comprehensive assessment of glycemic outcomes. In both the intensive care unit (ICU) and non-ICU setting, continuous glucose monitoring (CGM) has the potential to limit glycemic excursions by providing an early warning of impending hypo- or hyperglycemia, which may result in decreased morbidity and mortality.

\section{CGM Use in the ICU}

Hyperglycemia is a common problem affecting patients admitted to the intensive care unit. In the critically ill, hyperglycemia occurs not only in patients formally diagnosed with diabetes but can also be seen in patients without known diabetes; this group includes patients with undiagnosed type 2 diabetes and those with so-called stress hyperglycemia [13, 14]. At the same time, hypoglycemia and glucose variability have been identified as strong predictors of increased morbidity and mortality in the ICU [15]. It is often more difficult to detect hypoglycemia through the usual symptomatic signs or complaints in the ICU setting. Newer technologies such as CGMs can provide practitioners with not only more data points per day but additional useful glycemic information including direction and rate of glucose change. Due to a lack of sufficient accuracy in acutely ill patients, CGMs in the USA are currently approved in the outpatient setting only. Of note, during the current COVID-19 pandemic, the FDA temporarily instituted enforcement discretion allowing for expanded offlabel use of CGMs in the hospital for SARS-CoV-2 patients. $[16,17]$.

Most studies in ICU populations using CGM have focused on accuracy and reliability and have not been powered to detect changes in clinical outcomes $[2,10,18]$. Several concerns have been raised in regard to using CGM in the ICU population. Technological limitations that impede accuracy in subcutaneous continuous glucose sensors include buildup of tissue deposits (biofilm), the need for regular calibration due to sensor drift, measurement lag, and substance interference (acetaminophen, maltose, ascorbic acid, dopamine, mannitol, heparin, uric acid, and salicylic acid). There are also additional concerns regarding accuracy in critically ill patients with impaired tissue perfusion [19]. Of note, most of the studies included patients without diabetes, and few were performed in patients with type 1 diabetes, where glycemic excursions are more likely to occur [10].

Nevertheless, of the studies assessing glycemia in the inpatient setting, most did not show significant differences in average glycemic outcomes with CGM versus POC glucose testing [10]. An expert consensus meeting acknowledged that use of CGM in critical care populations appears to be accurate and reliable, but larger studies assessing clinical outcomes are needed [19]. Panel members also agreed that use of CGM at this time may not be feasible for every ICU patient. However, there are populations of interest who may benefit from further study of CGM because they are at high risk for glucose variability and hypoglycemia, and they include [1] any patients receiving insulin, especially intravenous insulin, [2] postcardiac surgery patients, [3] neonatal ICU patients, [4] posttransplant patients, [5] patients receiving glucocorticoids, [6] patients with end-stage renal or liver disease, [7] traumatic or vascular brain injury, and [8] those with hypoglycemia unawareness [19]. Similarly, the 2020 Continuous Glucose Monitor and Automated Insulin Dosing Systems in the Hospital Consensus Guideline Panel states that while bedside POC glucose has been historically the preferred method to assess glycemic management in the ICU, POC BG testing has some drawbacks. While CGM accuracy in ICU patients still needs to be validated, there remains a potential role for CGMs in ICU patients [3].

\section{CGM Use in the Non-ICU Setting}

A small number of studies have evaluated the use of CGM systems in the non-ICU setting. In 2013, Burt et al. published an observational study of 26 adult patients with diabetes (23 type 2 diabetes, 3 type 1 diabetes, $69 \%$ male, BMI $33 \pm 12 \mathrm{~kg} /$ $\mathrm{m}^{2}$ ), treated with basal-bolus insulin during hospitalization, using blinded CGMS System Gold in medical and surgical general wards in Australia [20]. There was no difference in the mean daily glucose values between CGM and POC capillary blood glucose testing $(172.8 \pm 43.2 \mathrm{mg} / \mathrm{dL}$ vs. $172.8 \pm$ $48.6 \mathrm{mg} / \mathrm{dL}$ with capillary testing, $P=.84$ ). Ten hypoglycemic episodes (glucose $<72 \mathrm{mg} / \mathrm{dL}$ ) occurred during the study duration, nine of which were exclusively detected with CGM. Two of these were severe hypoglycemic (glucose $<54 \mathrm{mg}$ / $\mathrm{dL}$ ) episodes. This observational study demonstrates the potential benefit of non-ICU CGM to increase hypoglycemia detection.

More recent studies comparing CGM interstitial glucose testing with capillary POC testing also reported that the mean glucose was similar between interstitial and capillary monitoring, but CGM testing detected a higher rate of hypoglycemic events $[18,21,22]$. While additional studies are needed to assess the impact of using CGMs on clinical outcomes, particularly in the T1D population, expert consensus recommends allowing continued use during hospitalizations if proper institutional protocols have been developed [19].

\section{Practical Considerations to Inpatient CGM Use}

The use of CGM offers several advantages over standard POC glucose testing in the inpatient setting. These advantages include increased frequency of glucose readings, data on glucose variability, greater rate of detection of hypoglycemia and hyperglycemia, possible alerts and alarms, and the potential 
for decreased nursing workload. Despite these advantages, limitations to inpatient use of CGM include lack of FDA approval, lack of widespread experience with this technology among general hospital practitioners, need for a remote monitoring and documentation infrastructure, and costs related to CGM supplies.

The 2020 Continuous Glucose Monitor and Automated Insulin Dosing Systems in the Hospital Consensus Guideline Panel recommends that hospital practitioners identify situations in which CGMs should not be used. These include (1) diabetic ketoacidosis, (2) situations with rapidly changing glucose levels and fluid/electrolyte shifts, (3) patients with skin infections or edema near the sensor site, and (4) patients treated with vasoactive agents or poor tissue perfusion [3].

It is important to keep in mind that most inpatient care team members will be unfamiliar with this technology. An institution interested in implementing CGM on the inpatient wards should consider having an interdisciplinary team with representation from physicians, nurse practitioners and/or physician assistants, diabetes care and education specialists, and nurses with diabetes technology expertise to help establish protocols and policies for CGM use [23]. This includes development of standard CGM data reports, workflows, and policies for when to perform POC testing to compare results to CGMs [3]. Having appropriate staff education/training and technical support would be key to safe implementation. The inpatient endocrinology or diabetes team will serve as an invaluable member of the patient's care team.

Patients already using CGMs in the outpatient setting should be advised to bring their supplies in from home. A single sensor can be worn for a limited period of time. The lifespan of the subcutaneous sensors depends on the specific manufacturer.

Another important aspect of effectively using CGM technology in the inpatient setting is to set up a remote monitoring station where glucose information is readily available to nursing staff and includes a system for automatic staff notification for CGM alarms [3]. A glucose telemetry system (GTS) can be implemented with three components: a CGM device, a smartphone (a phone with internet connectivity), and a tablet (e.g., iPad). Using commercially available software applications, glucose values are sent through Bluetooth or near-field communication (NFC) from the CGM transmitter to the smartphone, located next to the patient, and from there wirelessly to the tablet (or any other device with internet connection). The tablet can be located next to the clinician or at a staff work station and serves as a monitoring device presenting real-time CGM glucose values [23].

Additionally, safety precautions required with use during imaging studies (Table 2) and the potential for interference with glucose accuracy from medications and substances (acetaminophen, heparin, salicylic acid, dopamine, uric acid, ascorbic acid, maltose, mannitol, and tissue deposits) need to be taken into account [24]. Most CGM systems have not been tested during MRI, CT scans, or diathermy treatment (electric instruments generating heat); magnetic fields and heat could damage the components of the CGM, which may cause it to display inaccurate blood glucose readings or alerts. It is unknown if X-ray exposure during standard X-ray imaging studies will have an adverse effect. Table 2 reviews general recommendations from manufacturers regarding common radiologic studies. [25-27]. The Continuous Glucose Monitors and Automated Insulin Dosing Systems in the Hospital Consensus Guideline Panel recommends checking capillary or serum BG concentrations after procedures to ensure the patient's CGM is functioning properly [3].

Given the limitations of inpatient use of CGM discussed above, it is important to identify patients most likely to benefit from CGM use. Patients admitted with mild-to-moderate hyperglycemia and not on outpatient insulin therapy may be effectively treated with simple regimens requiring less frequent glucose monitoring [28]. However, patients with moderate to severe hyperglycemia require treatment with more complex insulin regimens. This group of patients include those with: (1) type 1 diabetes; (2) regimens of high-dose insulin or multiple non-insulin agents; (3) a long-standing history of diabetes; (4) significant glucotoxicity related to current infection, new diabetes diagnosis, or chronically uncontrolled hyperglycemia; or (5) iatrogenic hyperglycemia caused by high-dose steroid or medical nutrition therapy (enteral or parenteral nutrition) [28].

\section{The Hybrid Closed-Loop System}

Hybrid closed-loop systems, or automated insulin dosing (AID) systems, combine an insulin pump, a sensor for continuous glucose monitoring, and an automated algorithmic adjustment of basal insulin infusion rates and (in some models) correction boluses. Patient input on carbohydrate intake for bolusing and confirmatory fingerstick glucose calibration on some devices is still required. Initial studies evaluating the use of a closed-loop system in the hospital setting focused on the critical care setting or the peri-operative period. These small studies demonstrated good efficacy data with improvement in time in target glucose range and lower mean glucose levels without an increased risk of hypoglycemia [29-31].

Newer studies support the use of closed-loop insulin delivery with linked pump/sensor devices to manage blood glucose in select groups of hospitalized patients with type 2 diabetes [32-34]. Despite this evidence, the effect of closed-loop treatment on clinical outcomes, the best application of these devices, and cost-effectiveness of this approach are still to be determined [1]. The 2020 Continuous Glucose Monitors and Automated Insulin Dosing Systems in the Hospital Consensus Guideline Panel identifies various factors that may preclude 
their use in the inpatient setting [3]. The same contraindications to CSII discussed above apply to inpatient use of AID systems. The panel recommends only using AID systems in patients who have adequate knowledge and skills for using AID systems and that this decision should be periodically reassessed throughout the hospitalization. Hospital practitioners should also have an alternative plan for diabetes management in the event that the AID system is discontinued. As with inpatient use of CGMs, institutions need to establish protocols, order sets, and policies to implement AID systems safely. Hospital practitioners can consider switching AID systems from "auto" mode to "manual" mode in the inpatient setting, although this recommendation did not reach a consensus with the most recent panel meeting [3]. Given that a patient's condition can change rapidly in the inpatient setting, manual mode allows the inpatient practitioners to adjust insulin pump settings tailored to the condition of the patient, including glucose target range, insulin sensitivity factor, and basal rate, all of which are based on an algorithm in auto mode that has not been validated in acute care. For patient safety reasons, staying in auto mode may only be appropriate for short, straightforward hospitalizations.

Currently there are two hybrid closed-loop insulin pump systems approved for the outpatient setting: Medtronic's 670G and Tandem's Control-IQ. The closed-loop approach is an attractive option and can provide many benefits such as improved glycemic outcomes, reduced nursing workload, and improved patient satisfaction [24]. However, as with any novel technology, several limitations need to be addressed prior to wider adoption in the hospital setting. At this time, the decision to use hybrid closed-loop systems in the inpatient setting is limited to patients already using the system in the outpatient setting [3].

\section{Inpatient CGM Use in the SARS-CoV-2 Pandemic}

The rapid outbreak of novel coronavirus 2019 (COVID-19), which arose from severe acute respiratory syndrome coronavirus 2 (SARS-CoV-2) infection, has recently become an international public health emergency. Various cardiovascular and metabolic comorbidities, including diabetes, have been reported as a risk factor for unfavorable prognosis [35]. Anecdotal reports suggest that patients with severe COVID19 can present with marked hyperglycemia, even DKA, and can be very insulin resistant, whether or not there was a prior history of diabetes [36]. Given this information, maintaining optimal glucose levels in hospitalized patients is a key component of care. However, the need for multiple fingerstick glucose measurements per day contradicts efforts to conserve personal protective equipment (PPE) and reduce healthcare professionals' repeated exposure to the novel coronavirus and workload.

Some hospitals in the USA and around the world have turned to using CGMs to address this issue. On April 1, 2020, the FDA issued temporary allowances known as enforcement discretion to two CGM devices (Freestyle Libre [Abbott] and Dexcom G6 CGM) for in-hospital use for SARS-CoV-2 patients in response to the pandemic [17]. However, both device manufacturers recommend against using sensor data for making treatment decisions related to insulin therapy. The Continuous Glucose Monitors and Automated Insulin Dosing Systems in the Hospital Consensus Guideline Panel recognized that this unique situtation did not indicate "label approval" for CGM use in the hospital by regulatory bodies. Nevertheless, the panel agreed that clinicians should consider prescribing CGMs to reduce the need for frequent nurse contact for POC glucose testing and the use of PPE for patients on isolation with highly contagious infectious diseases [3].

CGMs are not currently widely used in the inpatient setting so if a decision is made by an institution to use CGM during an urgent scenario, there needs to be an awareness of the associated concerns and limitations (discussed above) before widespread implementation [37]. Anecdotally, institutions that started the process of utilizing CGM use during the COVID19 pandemic ran into issues with training, implementation, and resource utilization that not all hospitals were prepared for [3]. Additionally, institutions looking to expand the use of CGMs in the inpatient setting will need to have a protocol in place and a plan to purchase, store, clean, and stock CGM supplies, including transmitters, sensors, and receivers.

\section{Conclusion}

Continuous subcutaneous insulin infusion and continuous glucose monitoring play an important role in the treatment of diabetes. In the last decade, both CSII and CGM technology have been much more widely adopted in the ambulatory setting. There is consensus among experts and healthcare professional societies that inpatient use of CSII devices in carefully selected patients is safe and can improve inpatient glycemic management and reduce hypoglycemia.

The data on CGM use in the hospital setting is more limited, and larger studies assessing clinical outcomes are needed. Nevertheless, experts support continued use during hospitalizations if proper institutional protocols have been developed. More recently, CGMs played a vital role in managing hyperglycemia in hospitalized patients with COVID-19. CSII, CGMs, and AID systems offer tremendous promise in transforming glycemic management in hospitalized patients. 


\section{References}

1. Association AD. 15. Diabetes care in the hospital. Diabetes Care. 2020;43(Suppl 1):S193-202.

2. Umpierrez GE, Klonoff DC. Diabetes technology update: use of insulin pumps and continuous glucose monitoring in the hospital. Diabetes Care. 2018;41(8):1579-89.

3. Galindo RJ, Umpierrez GE, Rushakoff RJ, Basu A, Lohnes S, Nichols JH, et al. Continuous glucose monitors and automated insulin dosing systems in the hospital consensus guideline. $\mathrm{J}$ Diabetes Sci Technol 2020:1932296820954163.

4. Peters AL, Ahmann AJ, Battelino T, Evert A, Hirsch IB, Murad $\mathrm{MH}$, et al. Diabetes technology-continuous subcutaneous insulin infusion therapy and continuous glucose monitoring in adults: an Endocrine Society clinical practice guideline. J Clin Endocrinol Metab. 2016;101(11):3922-37.

5. Peters AL, Ahmann AJ, Hirsch IB, Raymond JK. Advances in glucose monitoring and automated insulin delivery: supplement to endocrine society clinical practice guidelines. J Endocr Soc. 2018;2(11):1214-25.

6. Thompson B, Leighton M, Korytkowski M, Cook CB. An overview of safety issues on use of insulin pumps and continuous glucose monitoring systems in the hospital. Curr Diab Rep. 2018;18(10):81.

7. Thompson B, Korytkowski M, Klonoff DC, Cook CB. Consensus statement on use of continuous subcutaneous insulin infusion therapy in the hospital. J Diabetes Sci Technol. 2018;12(4):880-9.

8. Cook CB, Beer KA, Seifert KM, Boyle ME, Mackey PA, Castro JC. Transitioning insulin pump therapy from the outpatient to the inpatient setting: a review of 6 years' experience with 253 cases. J Diabetes Sci Technol. 2012;6(5):995-1002.

9. Houlden RL, Moore S. In-hospital management of adults using insulin pump therapy. Can J Diabetes. 2014;38(2):126-33.

10. Davis GM, Galindo RJ, Migdal AL, Umpierrez GE. Diabetes Technology in the inpatient setting for management of hyperglycemia. Endocrinol Metab Clin N Am. 2020;49(1):79-93.

11. Levitt DL, Spanakis EK, Ryan KA, Silver KD. Insulin pump and continuous glucose monitor initiation in hospitalized patients with type 2 diabetes mellitus. Diabetes Technol Ther. 2018;20(1):32-8.

12. Kuzulugil D, Papeix G, Luu J, Kerridge RK. Recent advances in diabetes treatments and their perioperative implications. Curr Opin Anaesthesiol. 2019;32(3):398-404.

13. Carpenter DL, Gregg SR, Xu K, Buchman TG, Coopersmith CM. Prevalence and impact of unknown diabetes in the ICU. Crit Care Med. 2015;43(12):e541-50.

14. Honiden S, Inzucchi SE. Metabolic management during critical illness: glycemic control in the ICU. Semin Respir Crit Care Med. 2015;36(6):859-69.

15. Devanesan A, Lloyd J, Samad H, Saha S. Glycaemic control in intensive care: everything in moderation. J Intensive Care Soc. 2016;17(4):280-3.

16. Krinsley JS, Chase JG, Gunst J, Martensson J, Schultz MJ, Taccone FS, et al. Continuous glucose monitoring in the ICU: clinical considerations and consensus. Crit Care. 2017;21(1):197.

17. Administration USFaD. Coronavirus (COVID-19) Update: FDA allows expanded use of devices to monitor patients' vital signs remotely https://www.fda.gov/news-events/press-announcements/ coronavirus-covid-19-update-fda-allows-expanded-use-devicesmonitor-patients-vital-signs-remotely2020 [.

18. Levitt DL, Silver KD, Spanakis EK. Inpatient continuous glucose monitoring and glycemic outcomes. J Diabetes Sci Technol. 2017;11(5):1028-35.

19. Wallia A, Umpierrez GE, Rushakoff RJ, Klonoff DC, Rubin DJ, Hill Golden S, et al. The DTS continuous glucose monitoring in the hospital panel consensus statement on inpatient use of continuous glucose monitoring. J Diabetes Sci Technol. 2017;11(5):1036-44.

20. Burt MG, Roberts GW, Aguilar-Loza NR, Stranks SN. Brief report: comparison of continuous glucose monitoring and finger-prick blood glucose levels in hospitalized patients administered basalbolus insulin. Diabetes Technol Ther. 2013;15(3):241-5.

21. Schaupp L, Donsa K, Neubauer KM, Mader JK, Aberer F, Höll B, et al. Taking a closer look-continuous glucose monitoring in noncritically ill hospitalized patients with type 2 diabetes mellitus under basal-bolus insulin therapy. Diabetes Technol Ther. 2015;17(9): 611-8.

22. Gómez AM, Umpierrez GE, Muñoz OM, Herrera F, Rubio C, Aschner $\mathrm{P}$, et al. Continuous glucose monitoring versus capillary point-of-care testing for inpatient glycemic control in type 2 diabetes patients hospitalized in the general ward and treated with a basal bolus insulin regimen. J Diabetes Sci Technol. 2015;10(2):325-9.

23. Spanakis EK, Levitt DL, Siddiqui T, Singh LG, Pinault L, Sorkin J, et al. The effect of continuous glucose monitoring in preventing inpatient hypoglycemia in general wards: the glucose telemetry System. J Diabetes Sci Technol. 2018;12(1):20-5.

24. Wang M, Singh LG, Spanakis EK. Advancing the use of CGM devices in a non-ICU setting. J Diabetes Sci Technol. 2019;13(4): 674-81.

25. Inc. ADC. Freestyle Libre 14 day System User Manual. ART39764-001 2018 August 28, 2020. Available from: https:// freestyleserver.com/Payloads/IFU/2018/ART39764-001_rev-AWeb.pdf.

26. Dexcom I Dexcom G6 mobile continuous glucose monitoring system user guide. LBL014003 Rev 010 MT23976.2018 August 28, 2020. Available from: https://s3-us-west-2.ama-Available from: zonaws.com/dexcompdf/G6-CGM-Users-Guide.pdf.

27. Medtronic, Inc. Guardian Connect System User Guide. M995836A012 A. 2020 August 28, 2020. Available from: https://www.medtronicdiabetes.com/sites/default/files/library/ download-library/user-guides/Guardian-Connect-System-UserGuide.pdf.

28. Galindo RJ, Aleppo G, Klonoff DC, Spanakis EK, Agarwal S, Vellanki P, et al. Implementation of continuous glucose monitoring in the hospital: emergent considerations for remote glucose monitoring during the COVID-19 pandemic. J Diabetes Sci Technol. 2020;14(4):822-32.

29. Mibu K, Yatabe T, Hanazaki K. Blood glucose control using an artificial pancreas reduces the workload of ICU nurses. J Artif Organs. 2012;15(1):71-6.

30. Okabayashi T, Shima Y, Sumiyoshi T, Kozuki A, Tokumaru T, Iiyama $\mathrm{T}$, et al. Intensive versus intermediate glucose control in surgical intensive care unit patients. Diabetes Care. 2014;37(6): 1516-24.

31. Namikawa T, Munekage M, Kitagawa H, Yatabe T, Maeda H, Tsukamoto $\mathrm{Y}$, et al. Comparison between a novel and conventional artificial pancreas for perioperative glycemic control using a closedloop system. J Artif Organs. 2017;20(1):84-90.

32. Thabit H, Hartnell S, Allen JM, Lake A, Wilinska ME, Ruan Y, et al. Closed-loop insulin delivery in inpatients with type 2 diabetes: a randomised, parallel-group trial. Lancet Diabetes Endocrinol. 2017;5(2):117-24.

33. Bally L, Thabit H, Hartnell S, Andereggen E, Ruan Y, Wilinska $\mathrm{ME}$, et al. Closed-loop insulin delivery for glycemic control in noncritical care. N Engl J Med. 2018;379(6):547-56.

34. Boughton CK, Bally L, Martignoni F, Hartnell S, Herzig D, Vogt A, et al. Fully closed-loop insulin delivery in inpatients receiving nutritional support: a two-centre, open-label, randomised controlled trial. Lancet Diabetes Endocrinol. 2019;7(5):368-77.

35. Zhou F, Yu T, Du R, Fan G, Liu Y, Liu Z, et al. Clinical course and risk factors for mortality of adult inpatients with COVID-19 in 
Wuhan, China: a retrospective cohort study. Lancet. 2020;395(10229):1054-62.

36. Wake DJ, Gibb FW, Kar P, Kennon B, Klonoff DC, Rayman G, et al. Endocrinology in the time of COVID-19: Remodelling diabetes services and emerging innovation. Eur $\mathrm{J}$ Endocrinol. 2020;183(2):G67-77.

37. Korytkowski M, Antinori-Lent K, Drincic A, Hirsch IB, McDonnell ME, Rushakoff R, et al. A pragmatic approach to inpatient diabetes management during the COVID-19 pandemic. J Clin Endocrinol Metab. 2020;105(9):3076-87.

Publisher's Note Springer Nature remains neutral with regard to jurisdictional claims in published maps and institutional affiliations. 